\title{
On Shannon and Zipf-Mandelbrot entropies and related results
}

\author{
Sadia Khalid ${ }^{1 *}$, Đilda Pečarić ${ }^{2}$ and Josip Pečarić ${ }^{3}$
}

${ }^{\text {*Correspondence: }}$

saadiakhalid176@gmail.com;

skhalid@cuilahore.edu.pk

1 Department of Mathematics,

COMSATS University Islamabad,

Lahore, Pakistan

Full list of author information is

available at the end of the article

\begin{abstract}
In this paper, we present some interesting results related to the bounds of the Shannon and the Zipf-Mandelbrot entropies. Further, we define linear functionals and present their properties. We also construct new family of exponentially convex functions and Cauchy-type means.
\end{abstract}

MSC: 26A51;26D15; 52A41

Keywords: Shannon entropy; Zipf-Mandelbrot entropy; Non-increasing sequence in weighted mean; Wright-convex function; Cauchy means; $n$-exponential convexity and logarithmic convexity

\section{Introduction and preliminaries}

Definition 1.1 The Shannon entropy of a positive probability distribution $\mathbf{p}=\left(p_{1}, \ldots, p_{n}\right)$ is defined by $S(\mathbf{p}):=\sum_{k=1}^{n} p_{k} \log \left(\frac{1}{p_{k}}\right)$.

Definition 1.2 The Zipf-Mandelbrot law is a discrete probability distribution depending on three parameters $n \in \mathbb{N}, r \geq 0$ and $t>0$, and is defined as

$$
f(i ; n, r, t)=\frac{1}{(i+r)^{t} H_{n, r, t}}, \quad i \in\{1, \ldots, n\}
$$

where $f$ is known as the probability mass function and

$$
H_{n, r, t}:=\sum_{k=1}^{n} \frac{1}{(k+r)^{t}}
$$

is the generalized harmonic number.

If we take $p_{k}=\frac{1}{(k+r)^{t} H_{n, r} t}\left(1 \leq k \leq n, r \geq 0, t>0\right.$ and $H_{n, r, t}$ is the same as defined in Definition 1.2) in $S(\mathbf{p})$, then simple calculations reveal that

$$
\begin{aligned}
\sum_{k=1}^{n} \frac{1}{(k+r)^{t} H_{n, r, t}} \log \left((k+r)^{t} H_{n, r, t}\right) & =\frac{t}{H_{n, r, t}} \sum_{k=1}^{n} \frac{\log (k+r)}{(k+r)^{t}}+\log \left(H_{n, r, t}\right) \\
& :=Z\left(r, t, H_{n, r, t}\right)
\end{aligned}
$$

where $Z\left(r, t, H_{n, r, t}\right)$ is known as the Zipf-Mandelbrot entropy.

(c) The Author(s) 2019. This article is distributed under the terms of the Creative Commons Attribution 4.0 International License (http://creativecommons.org/licenses/by/4.0/), which permits unrestricted use, distribution, and reproduction in any medium, provided you give appropriate credit to the original author(s) and the source, provide a link to the Creative Commons license, and indicate if changes were made. 
A sequence $\left\{a_{k}\right\}_{k \in \mathbb{N}}$ of real numbers which is non-increasing in weighted mean (see [5]) can be defined as follows:

Definition 1.3 A sequence $\left\{a_{k}\right\}_{k \in \mathbb{N}} \subset \mathbb{R}$ is non-increasing in weighted mean if

$$
\frac{1}{P_{n}} \sum_{k=1}^{n} p_{k} a_{k} \geq \frac{1}{P_{n+1}} \sum_{k=1}^{n+1} p_{k} a_{k}, \quad n \in \mathbb{N}
$$

where $a_{k}$ and $p_{k}(k \in \mathbb{N})$ are real numbers such that $p_{i}>0(1 \leq i \leq k)$ with $P_{k}:=\sum_{i=1}^{k} p_{i}$ $(k \in \mathbb{N})$.

If the reversed inequality holds in (1.1), then the sequence $\left\{a_{k}\right\}_{k \in \mathbb{N}} \subset \mathbb{R}$ is called nondecreasing in weighted mean.

In a similar way, we can define when a finite sequence $\left\{a_{k}\right\}_{k=1}^{n} \subset \mathbb{R}$ is non-increasing or non-decreasing in weighted mean.

In [1] G. Bennett proved a weighted version of an inequality presented by HardyLittlewood-Pólya (see [2, Theorem 134]) for power functions $f(x)=x^{s}$ : if $a_{k}(1 \leq k \leq n)$ are non-negative and non-increasing and $p_{k} \geq 0$ for all $k \in\{1, \ldots, n\}$ such that $P_{k}=\sum_{i=1}^{k} p_{i}$ $(1 \leq k \leq n)$, then for any real number $s>1$, the inequality

$$
\left(\sum_{k=1}^{n} p_{k} a_{k}\right)^{s} \geq \sum_{k=1}^{n} P_{k}^{s}\left(a_{k}^{s}-a_{k+1}^{s}\right)=\left(p_{1} a_{1}\right)^{s}+\sum_{k=2}^{n} a_{k}^{s}\left(P_{k}^{s}-P_{k-1}^{s}\right)
$$

holds. If $0<s<1$, then the reversed inequality holds in (1.2) (see [1]).

S. Khalid, J. Pečarić and M. Praljak presented the following generalization of inequality (1.2) in [5, Theorem 3].

Theorem 1.4 Let $a_{k}$ and $p_{k}(1 \leq k \leq n)$ be real numbers such that $a_{k} \geq 0$ and $p_{k}>0$. Let $p_{1} a_{1}, \sum_{k=1}^{n} p_{k} a_{k}, P_{k} a_{k}, P_{k-1} a_{k} \in[a, b]$ for all $k \in\{2, \ldots, n\}$ and let $f:[a, b] \rightarrow \mathbb{R}$ be $a$ Wright-convex function.

(i) If the sequence $\left\{a_{k}\right\}_{k=1}^{n}$ is non-increasing in weighted mean, then

$$
f\left(\sum_{k=1}^{n} p_{k} a_{k}\right) \geq f\left(p_{1} a_{1}\right)+\sum_{k=2}^{n}\left[f\left(P_{k} a_{k}\right)-f\left(P_{k-1} a_{k}\right)\right] .
$$

(ii) If the sequence $\left\{a_{k}\right\}_{k=1}^{n}$ is non-decreasing in weighted mean, then

$$
f\left(\sum_{k=1}^{n} p_{k} a_{k}\right) \leq f\left(p_{1} a_{1}\right)+\sum_{k=2}^{n}\left[f\left(P_{k} a_{k}\right)-f\left(P_{k-1} a_{k}\right)\right] .
$$

Iff is Wright-concave, then the reversed inequalities hold in (1.3) and (1.4).

The results related to the Shannon entropy and the Zipf-Mandelbrot law are topic of great interest; see, for example, [3] and [7-10]. We present some interesting results related to the bounds of the Shannon entropy by using non-increasing (non-decreasing) sequence 
of real numbers and by applying Theorem 1.4. Further, we also present some results related to the bounds of the Zipf-Mandelbrot entropy. The Zipf-Mandelbrot law is revisited in the context of linguistics in [12] (see also [11]).

The paper is organized as follows: in Sects. 2 and 3, we present some interesting results related to the Shannon and the Zipf-Mandelbrot entropies, respectively. In Sect. 4, we define linear functionals as the non-negative differences of the obtained inequalities and present mean value theorems for the linear functionals. In Sect. 5, we present the properties of the functionals, such as $n$-exponential and logarithmic convexity. Finally, we give an example of the family of functions for which the results can be applied.

Remark 1.5 "log" denotes the logarithmic function and throughout this paper we consider the base $b$ of the logarithm to be greater than 1 .

\section{Inequalities related to the Shannon entropy}

In our first main result, we will use the following lemma:

\section{Lemma 2.1}

(i) If $p_{i} \in \mathbb{R}$ are such that $p_{i}>0(1 \leq i \leq n)$ and if the sequence $\left\{a_{i}\right\}_{i=1}^{n} \subset \mathbb{R}$ is non-increasing, then it is non-increasing in weighted mean.

(ii) If $p_{i} \in \mathbb{R}$ are such that $p_{i}>0(1 \leq i \leq n)$ and if the sequence $\left\{a_{i}\right\}_{i=1}^{n} \subset \mathbb{R}$ is non-decreasing, then it is non-decreasing in weighted mean.

Proof

(i) Simple calculations reveal that

$$
\frac{1}{P_{k}} \sum_{i=1}^{k} p_{i} a_{i}-\frac{1}{P_{k+1}} \sum_{i=1}^{k+1} p_{i} a_{i}=\frac{p_{k+1}}{P_{k} P_{k+1}}\left(\sum_{i=1}^{k} p_{i} a_{i}-P_{k} a_{k+1}\right) .
$$

As $a_{1} \geq \cdots \geq a_{n}$ and $p_{i}>0, i \in\{1, \ldots, n\}$, we have

$$
\begin{gathered}
p_{1} a_{1} \geq p_{1} a_{k+1}, \\
\vdots \\
p_{k} a_{k} \geq p_{k} a_{k+1} .
\end{gathered}
$$

Adding the above inequalities, we have $\sum_{i=1}^{k} p_{i} a_{i}-P_{k} a_{k+1} \geq 0$, which, together with $\frac{p_{k+1}}{P_{k} P_{k+1}}>0$, yields that $\frac{1}{P_{k}} \sum_{i=1}^{k} p_{i} a_{i} \geq \frac{1}{P_{k+1}} \sum_{i=1}^{k+1} p_{i} a_{i}$.

(ii) The proof is analogous to the proof of (i).

Our first main result is the following:

Theorem 2.2 Let $p_{k} \in \mathbb{R}$ such that $p_{k}>0(1 \leq k \leq n)$ and let $f:[a, b] \rightarrow \mathbb{R}$ be a Wrightconvex function.

(a) Let $0<p_{k}<1(1 \leq k \leq n)$ and let $S(\mathbf{p}), p_{1} \log \left(\frac{1}{p_{1}}\right), P_{k} \log \left(\frac{1}{p_{k}}\right), P_{k-1} \log \left(\frac{1}{p_{k}}\right)$ $\in[a, b]$ for all $k \in\{2, \ldots, n\}$. 
(i) If the sequence $\left\{p_{k}\right\}_{k=1}^{n}$ is non-increasing, then

$$
\begin{aligned}
f(S(\mathbf{p})) \leq & f\left(p_{1} \log \left(\frac{1}{p_{1}}\right)\right) \\
& +\sum_{k=2}^{n}\left[f\left(P_{k} \log \left(\frac{1}{p_{k}}\right)\right)-f\left(P_{k-1} \log \left(\frac{1}{p_{k}}\right)\right)\right] .
\end{aligned}
$$

(ii) If the sequence $\left\{p_{k}\right\}_{k=1}^{n}$ is non-decreasing, then

$$
\begin{aligned}
f(S(\mathbf{p})) \geq & f\left(p_{1} \log \left(\frac{1}{p_{1}}\right)\right) \\
& +\sum_{k=2}^{n}\left[f\left(P_{k} \log \left(\frac{1}{p_{k}}\right)\right)-f\left(P_{k-1} \log \left(\frac{1}{p_{k}}\right)\right)\right] .
\end{aligned}
$$

(b) Let $p_{k} \geq 1(1 \leq k \leq n)$ and let $-S(\mathbf{p}), p_{1} \log p_{1}, P_{k} \log p_{k}, P_{k-1} \log p_{k} \in[a, b]$ for all $k \in\{2, \ldots, n\}$.

(i) If the sequence $\left\{p_{k}\right\}_{k=1}^{n}$ is non-increasing, then

$$
f(-S(\mathbf{p})) \geq f\left(p_{1} \log p_{1}\right)+\sum_{k=2}^{n}\left[f\left(P_{k} \log p_{k}\right)-f\left(P_{k-1} \log p_{k}\right)\right]
$$

(ii) If the sequence $\left\{p_{k}\right\}_{k=1}^{n}$ is non-decreasing, then

$$
f(-S(\mathbf{p})) \leq f\left(p_{1} \log p_{1}\right)+\sum_{k=2}^{n}\left[f\left(P_{k} \log p_{k}\right)-f\left(P_{k-1} \log p_{k}\right)\right]
$$

Iff is Wright-concave, then the reversed inequalities hold in (2.1)-(2.4).

Proof

(a) (i) As $p_{1} \geq p_{2} \geq \cdots \geq p_{n}$ and $b>1$, the sequence $\left\{\log \left(\frac{1}{p_{k}}\right)\right\}_{k=1}^{n}$ is non-decreasing. By Lemma 2.1(ii), the sequence $\left\{\log \left(\frac{1}{p_{k}}\right)\right\}_{k=1}^{n}$ is non-decreasing in weighted mean and hence by using Theorem 1.4(ii) for $a_{k}=\log \left(\frac{1}{p_{k}}\right)$ such that $0<p_{k}<1$ $(1 \leq k \leq n)$, the result is immediate.

(ii) The idea of the proof is the same as discussed in (i).

(b) (i) As $p_{1} \geq p_{2} \geq \cdots \geq p_{n}$ and $b>1$, the sequence $\left\{\log p_{k}\right\}_{k=1}^{n}$ is non-increasing. By Lemma $2.1(\mathrm{i})$, the sequence $\left\{\log p_{k}\right\}_{k=1}^{n}$ is non-increasing in weighted mean and hence by taking $a_{k}=\log p_{k}$ with $p_{k} \geq 1(1 \leq k \leq n)$ in Theorem 1.4(i), the result is immediate.

(ii) The idea of the proof is the same as discussed in (i).

Since the class of convex (concave) functions is properly contained in the class of Wrightconvex (Wright-concave) functions, the following corollary is immediate.

Corollary 2.3 Let $p_{k} \in \mathbb{R}$ such that $p_{k}>0(1 \leq k \leq n)$ and let $f:[a, b] \rightarrow \mathbb{R}$ be a convex function. 
(a) Let all the conditions of Theorem 2.2(a) hold.

(i) If the sequence $\left\{p_{k}\right\}_{k=1}^{n}$ is non-increasing, then (2.1) holds.

(ii) If the sequence $\left\{p_{k}\right\}_{k=1}^{n}$ is non-decreasing, then (2.2) holds.

(b) Let all the conditions of Theorem 2.2(b) hold.

(i) If the sequence $\left\{p_{k}\right\}_{k=1}^{n}$ is non-increasing, then (2.3) holds.

(ii) If the sequence $\left\{p_{k}\right\}_{k=1}^{n}$ is non-decreasing, then (2.4) holds.

Iff is concave, then the reversed inequalities hold in (2.1)-(2.4).

An application of Corollary 2.3 is given as follows:

Corollary 2.4 Let $f(x)=x^{s}$, where $x \in(0, \infty)$ and $s \in \mathbb{R}$. Let $p_{k} \in \mathbb{R}$ such that $p_{k}>0(1 \leq$ $k \leq n)$.

(a) Let $0<p_{k}<1(1 \leq k \leq n)$ and let $s<0$ or $s>1$.

(i) If the sequence $\left\{p_{k}\right\}_{k=1}^{n}$ is non-increasing, then

$$
(S(\mathbf{p}))^{s} \leq\left(p_{1} \log \left(\frac{1}{p_{1}}\right)\right)^{s}+\sum_{k=2}^{n}\left(\log \left(\frac{1}{p_{k}}\right)\right)^{s}\left(P_{k}^{s}-P_{k-1}^{s}\right) .
$$

(ii) If the sequence $\left\{p_{k}\right\}_{k=1}^{n}$ is non-decreasing, then

$$
(S(\mathbf{p}))^{s} \geq\left(p_{1} \log \left(\frac{1}{p_{1}}\right)\right)^{s}+\sum_{k=2}^{n}\left(\log \left(\frac{1}{p_{k}}\right)\right)^{s}\left(P_{k}^{s}-P_{k-1}^{s}\right) .
$$

(b) Let $p_{k} \geq 1(1 \leq k \leq n)$ and let $s<0$ or $s>1$.

(i) If the sequence $\left\{p_{k}\right\}_{k=1}^{n}$ is non-increasing, then

$$
(-S(\mathbf{p}))^{s} \geq\left(p_{1} \log p_{1}\right)^{s}+\sum_{k=2}^{n}\left(\log p_{k}\right)^{s}\left(P_{k}^{s}-P_{k-1}^{s}\right) .
$$

(ii) If the sequence $\left\{p_{k}\right\}_{k=1}^{n}$ is non-decreasing, then

$$
(-S(\mathbf{p}))^{s} \leq\left(p_{1} \log p_{1}\right)^{s}+\sum_{k=2}^{n}\left(\log p_{k}\right)^{s}\left(P_{k}^{s}-P_{k-1}^{s}\right) .
$$

If $0<s<1$, then the reversed inequalities hold in (2.5)-(2.8).

\section{Inequalities related to the Zipf-Mandelbrot entropy}

The aim of this section is to present some interesting results by using the Zipf-Mandelbrot entropy.

Now, first we define the cumulative distribution function as follows:

$$
C_{k, n, r, t}:=\frac{H_{k, r, t}}{H_{n, r, t}}
$$

where $k \in\{1, \ldots, n\}, n \in \mathbb{N}, r \geq 0, t>0$ and $H_{n, r, t}$ is the same as defined in Definition 1.2.

The next result is the first main result of this section. 
Theorem 3.1 Let $Z\left(r, t, H_{n, r, t}\right)$ be the Zipf-Mandelbrot entropy, $C_{k, n, r, t}$ be the cumulative distribution function and $f:[a, b] \rightarrow \mathbb{R}$ be a Wright-convex function.

(i) Let $0<\frac{1}{(k+r)^{t} H_{n, r, t}}<1$. If $Z\left(r, t, H_{n, r, t}\right), \log \left((1+r)^{t} H_{n, r, t}\right)^{\frac{1}{(1+r)^{t} H_{n, r, t}}}$,

$$
\begin{aligned}
\log \left((k+r)^{t} H_{n, r, t}\right)^{C_{k, n, r, t}} & , \log \left((k+r)^{t} H_{n, r, t}\right)^{C_{k-1, n, r, t}} \in[a, b] \text { for all } k \in\{2, \ldots, n\}, \text { then } \\
f\left(Z\left(r, t, H_{n, r, t}\right)\right) \leq & f\left(\log \left((1+r)^{t} H_{n, r, t}\right)^{\frac{1}{(1+r)^{t} H_{n, r, t}}}\right) \\
& +\sum_{k=2}^{n} f\left(\log \left((k+r)^{t} H_{n, r, t}\right)^{C_{k, n, r, t}}\right) \\
& -\sum_{k=2}^{n} f\left(\log \left((k+r)^{t} H_{n, r, t}\right)^{C_{k-1, n, r, t}}\right) .
\end{aligned}
$$

(ii) Let $(k+r)^{t} H_{n, r, t} \leq 1$. If $-Z\left(r, t, H_{n, r, t}\right), \log \left((1+r)^{t} H_{n, r, t}\right)^{\frac{-1}{(1+r)^{t} H_{n, r, t}}}$, $\log \left((k+r)^{t} H_{n, r, t}\right)^{-C_{k, n, r, t}}, \log \left((k+r)^{t} H_{n, r, t}\right)^{-C_{k-1, n, r, t}} \in[a, b]$ for all $k \in\{2, \ldots, n\}$,

then

$$
\begin{aligned}
f\left(-Z\left(r, t, H_{n, r, t}\right)\right) \geq & f\left(\log \left((1+r)^{t} H_{n, r, t}\right)^{\frac{-1}{(1+r)^{t} H_{n, r, t}}}\right) \\
& +\sum_{k=2}^{n} f\left(\log \left((k+r)^{t} H_{n, r, t}\right)^{-C_{k, n, r, t}}\right) \\
& -\sum_{k=2}^{n} f\left(\log \left((k+r)^{t} H_{n, r, t}\right)^{-C_{k-1, n, r, t}}\right) .
\end{aligned}
$$

Iff is Wright-concave, then the reversed inequalities hold in (3.1) and (3.2).

Proof It is easy to see that the sequence $\left\{p_{k}=\frac{1}{(k+r)^{t} H_{n, r, t}}\right\}_{k=1}^{n}$ is non-increasing in $k \in$ $\{1, \ldots, n\}$.

(i) Taking $p_{k}=\frac{1}{(k+r)^{t} H_{n, r, t}}$ in Theorem 2.2(a)(i), the result is immediate.

(ii) The idea of the proof is the same as discussed in (i) but here we apply Theorem 2.2(b)(i) instead of Theorem 2.2(a)(i).

Corollary 3.2 Let $Z\left(r, t, H_{n, r, t}\right)$ be the Zipf-Mandelbrot entropy, $C_{k, n, r, t}$ be the cumulative distribution function and $f:[a, b] \rightarrow \mathbb{R}$ be a convex function.

(i) If all the conditions of Theorem 3.1(i) hold, then we have (3.1).

(ii) If all the conditions of Theorem 3.1(ii) hold, then we have (3.2).

Iff is concave, then the reversed inequalities hold in (3.1) and (3.2).

\section{Linear functionals and mean value theorems}

Consider inequalities (2.1), (2.3) and (3.1) and define linear functionals as follows:

$$
\begin{aligned}
\Phi_{1}(f)= & -f(S(\mathbf{p}))+f\left(p_{1} \log \left(\frac{1}{p_{1}}\right)\right) \\
& +\sum_{k=2}^{n}\left[f\left(P_{k} \log \left(\frac{1}{p_{k}}\right)\right)-f\left(P_{k-1} \log \left(\frac{1}{p_{k}}\right)\right)\right],
\end{aligned}
$$




$$
\begin{aligned}
\Phi_{2}(f)= & f(-S(\mathbf{p}))-f\left(p_{1} \log p_{1}\right) \\
& -\sum_{k=2}^{n}\left[f\left(P_{k} \log p_{k}\right)-f\left(P_{k-1} \log p_{k}\right)\right]
\end{aligned}
$$

and

$$
\begin{aligned}
\Phi_{3}(f)= & -f\left(Z\left(r, t, H_{n, r, t}\right)\right)+f\left(\log \left((1+r)^{t} H_{n, r, t}\right)^{\frac{1}{(1+r)^{t} H_{n, r, t}}}\right) \\
& +\sum_{k=2}^{n} f\left(\log \left((k+r)^{t} H_{n, r, t}\right)^{C_{k, n, r, t}}\right) \\
& -\sum_{k=2}^{n} f\left(\log \left((k+r)^{t} H_{n, r, t}\right)^{C_{k-1, n, r, t}}\right) .
\end{aligned}
$$

If $f$ is a convex function defined on $[a, b]$ and if the sequence $\left\{p_{k}\right\}_{k=1}^{n} \subset \mathbb{R}$ is nonincreasing, then Corollaries 2.3(a)(i) and 2.3(b)(i) imply that $\Phi_{1}(f) \geq 0$ and $\Phi_{2}(f) \geq 0$, respectively. Moreover, if $Z\left(r, t, H_{n, r, t}\right)$ is the Zipf-Mandelbrot entropy, $C_{k, n, r, t}$ is the cumulative distribution function and if $f:[a, b] \rightarrow \mathbb{R}$ is a convex function, then Corollary 3.2(i) implies that $\Phi_{3}(f) \geq 0$.

Now we present mean value theorems for the functional $\Phi_{i}(i=1, \ldots, 3)$. Lagrange-type mean value theorem related to $\Phi_{i}(i=1, \ldots, 3)$ is the following:

Theorem 4.1 Let $f:[a, b] \rightarrow \mathbb{R}$ be such that $f \in C^{2}([a, b])$ and let $\Phi_{1}, \Phi_{2}$ and $\Phi_{3}$ be the linear functionals as defined in (4.1), (4.2) and (4.3), respectively. Then there exists $\xi_{1}, \xi_{2}, \xi_{3} \in[a, b]$ such that

$$
\Phi_{i}(f)=\frac{f^{\prime \prime}\left(\xi_{i}\right)}{2} \Phi_{i}\left(f_{0}\right), \quad i \in\{1,2,3\}
$$

where $f_{0}(x)=x^{2}$.

Proof The proof is analogous to the proof of Theorem 2.7 given in [4] (see also Theorem 2.2 in [13]).

The following theorem is a new analogue of the classical Cauchy mean value theorem, related to $\Phi_{i}(i=1, \ldots, 3)$.

Theorem 4.2 Let $f, g:[a, b] \rightarrow \mathbb{R}$ be such that $f, g \in C^{2}([a, b])$ and let $\Phi_{1}, \Phi_{2}$ and $\Phi_{3}$ be the linear functionals as defined in (4.1), (4.2) and (4.3), respectively. Then there exist $\xi_{1}, \xi_{2}, \xi_{3} \in[a, b]$ such that

$$
\frac{\Phi_{i}(f)}{\Phi_{i}(g)}=\frac{f^{\prime \prime}\left(\xi_{i}\right)}{g^{\prime \prime}\left(\xi_{i}\right)}, \quad i \in\{1,2,3\},
$$

provided that the denominators are non-zero.

Proof The proof is analogous to the proof of Theorem 2.8 given in [4] (see also Theorem 2.4 in [13]). 


\section{Remark 4.3}

(i) By taking $f(x)=x^{s}$ and $g(x)=x^{q}$ in (4.4), where $s, q \in \mathbb{R} \backslash\{0,1\}$ are such that $s \neq q$, we have

$$
\xi_{i}^{s-q}=\frac{q(q-1) \Phi_{i}\left(x^{s}\right)}{s(s-1) \Phi_{i}\left(x^{q}\right)}, \quad i \in\{1,2,3\} .
$$

(ii) If the inverse of the function $f^{\prime \prime} / g^{\prime \prime}$ exists, then (4.4) implies that

$$
\xi_{i}=\left(\frac{f^{\prime \prime}}{g^{\prime \prime}}\right)^{-1}\left(\frac{\Phi_{i}(f)}{\Phi_{i}(g)}\right), \quad i \in\{1,2,3\} .
$$

\section{5 -exponential convexity and log-convexity}

In this section, first we will present a few important definitions, which will be useful further. In the sequel, let $I$ be an open interval in $\mathbb{R}$.

The next four definitions are given in [13].

Definition 5.1 A function $f: I \rightarrow \mathbb{R}$ is $n$-exponentially convex in the Jensen sense if

$$
\sum_{i, j=1}^{n} \varsigma_{i} \varsigma_{j} f\left(\frac{x_{i}+x_{j}}{2}\right) \geq 0
$$

holds for every $\varsigma_{i} \in \mathbb{R}$ and $x_{i} \in I(1 \leq i \leq n)$.

Definition 5.2 A function $f: I \rightarrow \mathbb{R}$ is $n$-exponentially convex if it is $n$-exponentially convex in the Jensen sense and continuous on $I$.

Definition 5.3 A function $f: I \rightarrow \mathbb{R}$ is exponentially convex in the Jensen sense if it $n$-exponentially convex in the Jensen sense for all $n \in \mathbb{N}$.

Definition 5.4 A function $f: I \rightarrow \mathbb{R}$ is exponentially convex if it is exponentially convex in the Jensen sense and continuous.

A log-convex function is defined as follows (see [14, p. 7]):

Definition 5.5 A function $f: I \rightarrow(0, \infty)$ is said to be $\log$-convex or multiplicatively convex if $\log f$ is convex. Equivalently, $f$ is $\log$-convex if for all $x, y \in I$ and for all $\lambda \in[0,1]$, the inequality

$$
f(\lambda x+(1-\lambda) y) \leq f^{\lambda}(x) f^{(1-\lambda)}(y)
$$

holds. If the inequality reverses, then $f$ is said to be log-concave.

Divided difference of a function is defined as follows (see [14, p. 14]):

Definition 5.6 The $n$ th-order divided difference of a function $f:[a, b] \rightarrow \mathbb{R}$ at mutually distinct points $x_{0}, \ldots, x_{n} \in[a, b]$ is defined recursively by

$$
\left[x_{i} ; f\right]=f\left(x_{i}\right), \quad i \in\{0, \ldots, n\} \text {, }
$$




$$
\left[x_{0}, \ldots, x_{n} ; f\right]=\frac{\left[x_{1}, \ldots, x_{n} ; f\right]-\left[x_{0}, \ldots, x_{n-1} ; f\right]}{x_{n}-x_{0}} .
$$

The value $\left[x_{0}, \ldots, x_{n} ; f\right]$ is independent of the order of the points $x_{0}, \ldots, x_{n}$.

The $n$-convex functions can be characterized by the $n$ th-order divided difference (see $[14$, p. 15]).

Definition 5.7 A function $f:[a, b] \rightarrow \mathbb{R}$ is said to be $n$-convex $(n \geq 0)$ if and only if for all choices of $(n+1)$ distinct points $x_{0}, \ldots, x_{n} \in[a, b]$, the $n$ th-order divided difference of $f$ satisfies $\left[x_{0}, \ldots, x_{n} ; f\right] \geq 0$.

Remark 5.8 Note that 0-convex functions are non-negative functions, 1-convex functions are increasing functions, and 2-convex functions are simply the convex functions.

Next we study the $n$-exponential convexity and log-convexity of the functions associated with the linear functionals $\Phi_{i}(i=1, \ldots, 3)$ as defined in (4.1)-(4.3).

Theorem 5.9 Let $\Omega=\left\{f_{s}: s \in I \subseteq \mathbb{R}\right\}$ be a family of functions defined on $[a, b]$ such that the function $s \mapsto\left[z_{0}, z_{1}, z_{2} ; f_{s}\right]$ is n-exponentially convex in the Jensen sense on I for every three mutually distinct points $z_{0}, z_{1}, z_{2} \in[a, b]$. Let $\Phi_{i}(i=1, \ldots, 3)$ be the linear functionals as defined in (4.1)-(4.3). Then the following statements hold:

(i) The function $s \mapsto \Phi_{i}\left(f_{s}\right)$ is n-exponentially convex in the Jensen sense on I and the matrix $\left[\Phi_{i}\left(\frac{f_{s_{j}+s_{k}}}{2}\right)\right]_{j, k=1}^{m}$ is positive semi-definite for all $m \in \mathbb{N}, m \leq n$ and $s_{1}, \ldots, s_{m} \in$ I. In particular,

$$
\operatorname{det}\left[\Phi_{i}\left(f_{\frac{s_{j}+s_{k}}{2}}\right)\right]_{j, k=1}^{m} \geq 0, \quad \forall m \in \mathbb{N}, m \leq n
$$

(ii) If the function $s \mapsto \Phi_{i}\left(f_{s}\right)$ is continuous on $I$, then it is n-exponentially convex on I.

Proof The idea of the proof is the same as that of the proof of Theorem 9 in [5].

The following corollary is an immediate consequence of Theorem 5.9.

Corollary 5.10 Let $\Omega=\left\{f_{s}: s \in I \subseteq \mathbb{R}\right\}$ be a family of functions defined on $[a, b]$ such that the function $s \mapsto\left[z_{0}, z_{1}, z_{2} ; f_{s}\right]$ is exponentially convex in the Jensen sense on I for every three mutually distinct points $z_{0}, z_{1}, z_{2} \in[a, b]$. Let $\Phi_{i}(i=1, \ldots, 3)$ be the linear functionals as defined in (4.1)-(4.3). Then the following statements hold:

(i) The function $s \mapsto \Phi_{i}\left(f_{s}\right)$ is exponentially convex in the Jensen sense on I and the matrix $\left[\Phi_{i}\left(\frac{f_{s+s_{k}}}{2}\right)\right]_{j, k=1}^{m}$ is positive semi-definite for all $m \in \mathbb{N}, m \leq n$ and $s_{1}, \ldots, s_{m} \in I$. In particular,

$$
\operatorname{det}\left[\Phi_{i}\left(\frac{f_{s_{j}+s_{k}}}{2}\right)\right]_{j, k=1}^{m} \geq 0, \quad \forall m \in \mathbb{N}, m \leq n
$$

(ii) If the function $s \mapsto \Phi_{i}\left(f_{s}\right)$ is continuous on $I$, then it is exponentially convex on $I$. 
Corollary 5.11 Let $\Omega=\left\{f_{s}: s \in I \subseteq \mathbb{R}\right\}$ be a family of functions defined on $[a, b]$ such that the function $s \mapsto\left[z_{0}, z_{1}, z_{2} ; f_{s}\right]$ is 2-exponentially convex in the Jensen sense on I for every three mutually distinct points $z_{0}, z_{1}, z_{2} \in[a, b]$. Let $\Phi_{i}(i=1, \ldots, 3)$ be the linear functionals as defined in (4.1)-(4.3). Further, assume that $\Phi_{i}\left(f_{s}\right)(i=1, \ldots, 3)$ is strictly positive for $f_{s} \in \Omega$. Then the following statements hold:

(i) If the function $s \mapsto \Phi_{i}\left(f_{s}\right)$ is continuous on I, then it is 2-exponentially convex on I and so it is $\log$-convex on I and for $\tilde{r}, s, \tilde{t} \in I$ such that $\tilde{r}<s<\tilde{t}$, we have

$$
\left[\Phi_{i}\left(f_{s}\right)\right]^{\tilde{t}-\tilde{r}} \leq\left[\Phi_{i}\left(f_{\tilde{r}}\right)\right]^{\tilde{t}-s}\left[\Phi_{i}\left(f_{\tilde{t}}\right)\right]^{s-\tilde{r}}, \quad i \in\{1,2,3\}
$$

which is known as Lyapunov's inequality. If $\tilde{r}<\tilde{t}<s$ or $s<\tilde{r}<\tilde{t}$, then the reversed inequality holds in (5.1).

(ii) If the function $s \mapsto \Phi_{i}\left(f_{s}\right)$ is differentiable on I, then for every $s, q, u, v \in I$ such that $s \leq u$ and $q \leq v$, we have

$$
\mu_{s, q}\left(\Phi_{i}, \Omega\right) \leq \mu_{u, v}\left(\Phi_{i}, \Omega\right), \quad i \in\{1,2,3\}
$$

where

$$
\mu_{s, q}\left(\Phi_{i}, \Omega\right)= \begin{cases}\left(\frac{\Phi_{i}\left(f_{s}\right)}{\Phi_{i}\left(f_{q}\right)}\right)^{\frac{1}{s-q}}, & s \neq q, \\ \exp \left(\frac{\frac{d}{d s} \Phi_{i}\left(f_{s}\right)}{\Phi_{i}\left(f_{s}\right)}\right), & s=q,\end{cases}
$$

for $f_{s}, f_{q} \in \Omega$.

Proof The idea of the proof is the same as that of the proof of Corollary 5 given in [5].

Remark 5.12 Note that the results from Theorem 5.9, as well as Corollaries 5.10 and 5.11 still hold when two of the points $z_{0}, z_{1}, z_{2} \in[a, b]$ coincide, say $z_{1}=z_{0}$, for a family of differentiable functions $f_{s}$ such that the function $s \mapsto\left[z_{0}, z_{1}, z_{2} ; f_{s}\right]$ is $n$-exponentially convex in the Jensen sense (exponentially convex in the Jensen sense, log-convex in the Jensen sense on $I$ ); and furthermore, they still hold when all three points coincide for a family of twice differentiable functions with the same property.

There are several families of functions which fulfil the conditions of Theorem 5.9, Corollaries 5.10 and 5.11, and Remark 5.12 and so the results of these theorem and corollaries can be applied to them. Here we present an example for such a family of functions; for more examples see [6].

Example 5.13 Consider the family of functions

$$
\tilde{\Omega}=\left\{f_{s}:(0, \infty) \rightarrow \mathbb{R}: s \in \mathbb{R}\right\}
$$

defined by

$$
f_{s}(x)= \begin{cases}\frac{x^{s}}{s(s-1)}, & s \notin\{0,1\}, \\ -\log x, & s=0, \\ x \log x, & s=1 .\end{cases}
$$


Here $\frac{d^{2}}{d x^{2}} f_{s}(x)=x^{s-2}=e^{(s-2) \log x}>0$, which shows that $f_{s}$ is convex for $x>0$ and $s \mapsto$ $\frac{d^{2}}{d x^{2}} f_{s}(x)$ is exponentially convex by definition.

In order to prove that the function $s \mapsto\left[z_{0}, z_{1}, z_{2} ; f_{s}\right]$ is exponentially convex, it is enough to show that

$$
\sum_{j, k=1}^{n} \varsigma_{j} \varsigma_{k}\left[z_{0}, z_{1}, z_{2} ; \frac{f_{\frac{s_{j}+s_{k}}{2}}}{2}\right]=\left[z_{0}, z_{1}, z_{2} ; \sum_{j, k=1}^{n} \varsigma_{j} \varsigma_{k} \frac{f_{j_{j}+s_{k}}}{2}\right] \geq 0
$$

for all $n \in \mathbb{N}, \varsigma_{j}, s_{j} \in \mathbb{R}, j \in\{1, \ldots, n\}$. By Definition 5.7, inequality (5.4) will hold if $\Xi(x):=$ $\sum_{j, k=1}^{n} \varsigma_{j} \varsigma_{k} f_{\frac{s_{j}+s_{k}}{2}}(x)$ is convex. Since $s \mapsto \frac{d^{2}}{d x^{2}} f_{s}(x)$ is exponentially convex, that is,

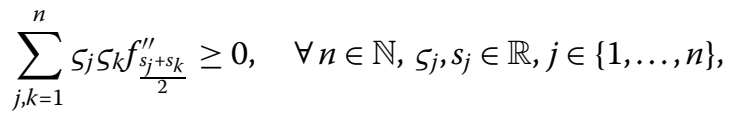

which shows the convexity of $\Xi$, inequality (5.4) is immediate. Now as the function $s \mapsto\left[z_{0}, z_{1}, z_{2} ; f_{s}\right]$ is exponentially convex, $s \mapsto\left[z_{0}, z_{1}, z_{2} ; f_{s}\right]$ is exponentially convex in the Jensen sense and, by using Corollary 5.10, we have $s \mapsto \Phi_{i}\left(f_{s}\right)(i=1, \ldots, 3)$ is exponentially convex in the Jensen sense. Since these mappings are continuous, $s \mapsto \Phi_{i}\left(f_{s}\right)(i=1, \ldots, 3)$ is exponentially convex.

If $\tilde{r}, s, \tilde{t} \in \mathbb{R}$ are such that $\tilde{r}<s<\tilde{t}$, then from (5.1) we have

$$
\Phi_{i}\left(f_{s}\right) \leq\left[\Phi_{i}\left(f_{\tilde{r}}\right)\right]^{\frac{\tilde{t}-s}{\tilde{t}-\tilde{r}}}\left[\Phi_{i}\left(f_{\tilde{t}}\right)\right]^{\frac{s-\tilde{r}}{\tilde{t}-\tilde{r}}}, \quad i \in\{1,2,3\}
$$

If $\tilde{r}<\tilde{t}<s$ or $s<\tilde{r}<\tilde{t}$, then the reversed inequality holds in (5.5).

Particularly for $i \in\{1,2,3\}$ and $\tilde{r}, s, \tilde{t} \in \mathbb{R} \backslash\{0,1\}$ such that $\tilde{r}<s<\tilde{t}$, we have

$$
\begin{aligned}
& \frac{-S^{s}(\mathbf{p})+\left(p_{1} \log \left(\frac{1}{p_{1}}\right)\right)^{s}+\sum_{k=2}^{n}\left(\log \left(\frac{1}{p_{k}}\right)\right)^{s}\left(P_{k}^{s}-P_{k-1}^{s}\right)}{s(s-1)} \\
& \leq\left[\frac{-S^{\tilde{r}}(\mathbf{p})+\left(p_{1} \log \left(\frac{1}{p_{1}}\right)\right)^{\tilde{r}}+\sum_{k=2}^{n}\left(\log \left(\frac{1}{p_{k}}\right)\right)^{\tilde{r}}\left(P_{k}^{\tilde{r}}-P_{k-1}^{\tilde{r}}\right)}{\tilde{r}(\tilde{r}-1)}\right]^{\tilde{\tilde{t}-s}} \\
& \times\left[\frac{-S^{\tilde{t}}(\mathbf{p})+\left(p_{1} \log \left(\frac{1}{p_{1}}\right)\right)^{\tilde{t}}+\sum_{k=2}^{n}\left(\log \left(\frac{1}{p_{k}}\right)\right)^{\tilde{t}}\left(P_{k}^{\tilde{t}}-P_{k-1}^{\tilde{t}}\right)}{\tilde{t}(\tilde{t}-1)}\right]^{\frac{s-\tilde{r}}{\tilde{t} \tilde{r}}}, \\
& \frac{(-S(\mathbf{p}))^{s}-\left(p_{1} \log p_{1}\right)^{s}-\sum_{k=2}^{n}\left(\log p_{k}\right)^{s}\left(P_{k}^{s}-P_{k-1}^{s}\right)}{s(s-1)} \\
& \leq\left[\frac{(-S(\mathbf{p}))^{\tilde{r}}-\left(p_{1} \log p_{1}\right)^{\tilde{r}}-\sum_{k=2}^{n}\left(\log p_{k}\right)^{\tilde{r}}\left(P_{k}^{\tilde{r}}-P_{k-1}^{\tilde{r}}\right)}{\tilde{r}(\tilde{r}-1)}\right]^{\tilde{t}-\tilde{r}} \\
& \times\left[\frac{(-S(\mathbf{p}))^{\tilde{t}}-\left(p_{1} \log p_{1}\right)^{\tilde{t}}-\sum_{k=2}^{n}\left(\log p_{k}\right)^{\tilde{t}}\left(P_{k}^{\tilde{t}}-P_{k-1}^{\tilde{t}}\right)}{\tilde{t}(\tilde{t}-1)}\right]^{\frac{s-\tilde{r}}{\tilde{t}-\tilde{r}}}
\end{aligned}
$$


and

$$
\begin{aligned}
& \frac{1}{s(s-1)}\left[-Z^{s}\left(r, t, H_{n, r, t}\right)+\left(\log \left((1+r)^{t} H_{n, r, t}\right)^{\frac{1}{(1+r)^{t} H_{n, r} t}}\right)^{s}\right. \\
& \left.+\sum_{k=2}^{n}\left[\left(\log \left((k+r)^{t} H_{n, r, t}\right)^{C_{k, n, r, t}}\right)^{s}-\left(\log \left((k+r)^{t} H_{n, r, t}\right)^{C_{k-1, n, r, t}}\right)^{s}\right]\right] \\
& \leq\left(\frac{1}{\tilde{r}(\tilde{r}-1)}\right)^{\frac{\tilde{t}-s}{\tilde{t}-\tilde{r}}}\left[-Z^{\tilde{r}}\left(r, t, H_{n, r, t}\right)+\left(\log \left((1+r)^{t} H_{n, r, t}\right)^{\left.\frac{1}{(1+r)^{t} H_{n, r, t}}\right)^{\tilde{r}}}\right.\right. \\
& \left.+\sum_{k=2}^{n}\left[\left(\log \left((k+r)^{t} H_{n, r, t}\right)^{C_{k, n, r, t}}\right)^{\tilde{r}}-\left(\log \left((k+r)^{t} H_{n, r, t}\right)^{C_{k-1, n, r, t}}\right)^{\tilde{r}}\right]\right] \\
& \quad \times\left(\frac{1}{\tilde{t}(\tilde{t}-1)}\right)^{\frac{s-\tilde{\tau}}{\tilde{t}-\tilde{r}}}\left[-Z^{\tilde{t}}\left(r, t, H_{n, r, t}\right)+\left(\log \left((1+r)^{t} H_{n, r, t}\right)^{\frac{\tilde{t}-s}{\tilde{t} \tilde{r}}}\right.\right. \\
& \left.\quad+\sum_{k=2}^{n}\left[\left(\log \left((k+r)^{t} H_{n, r, t}\right)^{C_{k, n, r, t}}\right)^{\tilde{t}}-\left(\log \left((k+r)^{t} H_{n, r, t}\right)^{C_{k-1, n, r, t}}\right)^{\tilde{t}}\right]\right]^{\tilde{t}} .
\end{aligned}
$$

In this case, $\mu_{s, q}\left(\Phi_{i}, \Omega\right)(i=1, \ldots, 3)$ defined in (5.3) becomes

$$
\mu_{s, q}\left(\Phi_{i}, \tilde{\Omega}\right)= \begin{cases}\left(\frac{\Phi_{i}\left(f_{s}\right)}{\Phi_{i}\left(f_{q}\right)}\right)^{\frac{1}{s-q}}, & s \neq q, \\ \exp \left(\frac{1-2 s}{s(s-1)}-\frac{\Phi_{i}\left(f_{0} f_{s}\right)}{\Phi_{i}\left(f_{s}\right)}\right), & s=q \notin\{0,1\}, \\ \exp \left(1-\frac{\Phi_{i}\left(f_{0}^{2}\right)}{2 \Phi_{i}\left(f_{0}\right)}\right), & s=q=0, \\ \exp \left(-1-\frac{\Phi_{i}\left(f_{0} f_{1}\right)}{2 \Phi_{i}\left(f_{1}\right)}\right), & s=q=1 .\end{cases}
$$

In particular for $i=1$, we have

$$
\begin{aligned}
\Phi_{1}\left(f_{s}\right)= & \frac{1}{s(s-1)}\left[-S^{s}(\mathbf{p})+p_{1}^{s} \log ^{s}\left(\frac{1}{p_{1}}\right)+\sum_{k=2}^{n} \log ^{s}\left(\frac{1}{p_{k}}\right)\left(P_{k}^{s}-P_{k-1}^{s}\right)\right], \\
& s \notin\{0,1\}, \\
\Phi_{1}\left(f_{0}\right)= & \log \left(\frac{S(\mathbf{p})}{p_{1} \log \left(\frac{1}{p_{1}}\right)}\right)+\sum_{k=2}^{n} \log \left(\frac{P_{k-1}}{P_{k}}\right), \\
\Phi_{1}\left(f_{1}\right)= & \log \left(\frac{\left(p_{1} \log \left(\frac{1}{p_{1}}\right)\right)^{p_{1} \log \left(\frac{1}{p_{1}}\right)}}{(S(\mathbf{p}))^{S(\mathbf{p})}}\right) \\
& +\sum_{k=2}^{n} \log \left(\frac{\left(P_{k} \log \left(\frac{1}{p_{k}}\right)\right)^{P_{k} \log \left(\frac{1}{p_{k}}\right)}}{\left.\left(P_{k-1} \log \left(\frac{1}{p_{k}}\right)\right)^{P_{k-1} \log \left(\frac{1}{p_{k}}\right)}\right)}\right. \\
\Phi_{1}\left(f_{0}^{2}\right)= & \sum_{k=2}^{n}\left[\log ^{2}\left(P_{k} \log \left(\frac{1}{p_{k}}\right)\right)-\log ^{2}\left(P_{k-1} \log \left(\frac{1}{p_{k}}\right)\right)\right]
\end{aligned}
$$




$$
\begin{aligned}
& +\log ^{2}\left(p_{1} \log \left(\frac{1}{p_{1}}\right)\right)-\log ^{2}(S(\mathbf{p})), \\
\Phi_{1}\left(f_{0} f_{1}\right)= & S(\mathbf{p}) \log ^{2}(S(\mathbf{p}))-p_{1} \log \left(\frac{1}{p_{1}}\right) \log ^{2}\left(p_{1} \log \left(\frac{1}{p_{1}}\right)\right) \\
& -\sum_{k=2}^{n} \log \left(\frac{1}{p_{k}}\right) P_{k} \log ^{2}\left(P_{k} \log \left(\frac{1}{p_{k}}\right)\right) \\
& +\sum_{k=2}^{n} \log \left(\frac{1}{p_{k}}\right) P_{k-1} \log ^{2}\left(P_{k-1} \log \left(\frac{1}{p_{k}}\right)\right)
\end{aligned}
$$

and

$$
\begin{aligned}
\Phi_{1}\left(f_{0} f_{s}\right)= & \frac{1}{s(s-1)} \log \left(\frac{(S(\mathbf{p}))^{(s(\mathbf{p}))^{s}}}{\left(p_{1} \log \left(\frac{1}{p_{1}}\right)\right)^{p_{1}^{s} \log ^{s}\left(\frac{1}{p_{1}}\right)}}\right) \\
& +\frac{1}{s(s-1)} \sum_{k=2}^{n} \log \left(\frac{\left(P_{k-1} \log \left(\frac{1}{p_{k}}\right)\right)^{P_{k-1}^{s} \log ^{s}\left(\frac{1}{p_{k}}\right)}}{\left(P_{k} \log \left(\frac{1}{p_{k}}\right)\right)^{\left(P_{k}^{s} \log ^{s}\left(\frac{1}{p_{k}}\right)\right)^{s}}}\right), \quad s \notin\{0,1\} .
\end{aligned}
$$

If $\Phi_{i}(i=1, \ldots, 3)$ is positive, then Theorem 4.2 applied for $f=f_{s} \in \tilde{\Omega}$ and $g=f_{q} \in \tilde{\Omega}$ yields that there exists $\xi_{i} \in[a, b]$ such that

$$
\xi_{i}^{s-q}=\frac{\Phi_{i}\left(f_{s}\right)}{\Phi_{i}\left(f_{q}\right)}, \quad i \in\{1,2,3\}
$$

Since the function $\xi_{i} \mapsto \xi_{i}^{s-q}$ is invertible for $s \neq q$, we have

$$
a \leq\left(\frac{\Phi_{i}\left(f_{s}\right)}{\Phi_{i}\left(f_{q}\right)}\right)^{\frac{1}{s-q}} \leq b, \quad i \in\{1,2,3\},
$$

which, together with the fact that $\mu_{s, q}\left(\Phi_{i}, \tilde{\Omega}\right)(i=1, \ldots, 3)$ is continuous, symmetric and monotonous (by (5.2)), shows that $\mu_{s, q}\left(\Phi_{i}, \tilde{\Omega}\right)$ is a mean.

\section{Acknowledgements}

The research of the 3rd author is supported by the Ministry of Education and Science of the Russian Federation (Agreement No. 02.a03.21.0008).

Funding

Not applicable.

Availability of data and materials

Not applicable.

\section{Competing interests}

The authors declare that they have no competing interests.

\section{Authors' contributions}

All authors contributed to this paper equally and all read and approved the final manuscript.

\section{Author details}

${ }^{1}$ Department of Mathematics, COMSATS University Islamabad, Lahore, Pakistan. ${ }^{2}$ Catholic University of Croatia, Zagreb,

Croatia. ${ }^{3}$ Rudn University, Moscow, Russia. 


\section{Publisher's Note}

Springer Nature remains neutral with regard to jurisdictional claims in published maps and institutional affiliations.

Received: 1 December 2018 Accepted: 21 March 2019 Published online: 11 April 2019

\section{References}

1. Bennett, G.: Lower bounds for matrices. Linear Algebra Appl. 82, $81-98$ (1986)

2. Hardy, G.H., Littlewood, J.E., Pólya, G.: Inequalities, 2nd edn. Cambridge University Press, Cambridge (1952)

3. Jakšetić, J., Pečarić, Đ., Pečarić, J.: Some properties of Zipf-Mandelbrot law and Hurwitz $\xi$-function. Math. Inequal. Appl. 21, 575-584 (2018)

4. Khalid, S., Pečarić, J., Praljak, M.: 3-convex functions and generalizations of an inequality of Hardy-Littlewood-Pólya. Glas. Mat. Ser. III 48(68), 335-356 (2013)

5. Khalid, S., Pečarić, J., Praljak, M.: On an inequality of I. Perić. Math. Commun. 19, 221-242 (2014)

6. Khalid, S., Pečarić, J., Vukelić, A.: Refinements of the majorization theorems via Fink identity and related results. J. Class. Anal. 7(2), 129-154 (2015)

7. Khan, M.A., Al-Sahwi, Z.M., Chu, Y.M.: New estimations for Shannon and Zipf-Mandelbrot entropies. Entropy 20(8), 608 (2018)

8. Khan, M.A., Pečarić, Đ., Pečarić, J.: Bounds for Shannon and Zipf-Mandelbrot entropies. Math. Methods Appl. Sci. 40(18), 7316-7322 (2017)

9. Khan, M.A., Pečarić, Đ., Pečarić, J.: On Zipf-Mandelbrot entropy. J. Comput. Appl. Math. 346, 192-204 (2019)

10. Latif, N., Pečarić, Đ., Pečarić, J.: Majorization, Csiszar divergence and Zipf-Mandelbrot law. J. Inequal. Appl. 2017, 197 (2017)

11. Mann, M.G., Tsallis, C.: Nonextensive Entropy: Interdisciplinary Applications. Oxford University Press, London (2004)

12. Montemurro, M.A.: Beyond the Zipf-Mandelbrot law in quantitative linguistics. Physica A 300, 567-578 (2001)

13. Pečarić, J., Perić, J.: Improvements of the Giaccardi and the Petrović inequality and related Stolarsky type means. An. Univ. Craiova, Ser. Mat. Inform. 39, 65-75 (2012)

14. Pečarić, J., Proschan, F., Tong, Y.L.: Convex Functions, Partial Orderings, and Statistical Applications. Academic Press, San Diego (1992)

\section{Submit your manuscript to a SpringerOpen ${ }^{\circ}$ journal and benefit from:}

- Convenient online submission

- Rigorous peer review

- Open access: articles freely available online

- High visibility within the field

- Retaining the copyright to your article

Submit your next manuscript at $\boldsymbol{\Delta}$ springeropen.com 